\title{
PARAMETER CHARACTERIZATION AND DEVELOPMENT OF ARTIFICIAL NEURAL NETWORK INVERSE MODEL OF A PM DIRECT CURRENT MOTOR
}

OKAFOR P.U., ARINZE S.N., OFFIA I.S.

Department of Electrical and Electronic Engineering Department, Faculty of Engineering, Enugu State University of Science and Technology (ESUT), Enugu State, Nigeria

\begin{abstract}
Direct Current (DC) motors are used extensively in control systems especially in industrial actuators. Thus, it is paramount to establish mathematical model for analysis, efficient control and application of DC motors. DC motor takes in single input in the form of an input voltage and generates a single output in the form of output speed. The motor parameters were characterized to understand the behavior of the motor. Artificial neural network (ANN) which is one of the newest signal-processing technologies in the engineer's toolbox was used as the modeling tool. Mathematical model representing a single transfer function of the DC motor was developed and hence, the discrete form of the motor .The system performance was improved by integration of the speed and position profiles as a single control system.
\end{abstract}

Keywords: ANN, Control, DC Motor, MRAC, Position, Speed.

\subsection{Introduction}

The complexity of high performance drives used in automated processes such as production lines, conveying systems etc., is increasing exponentially. Therefore, there is need to achieve greater efficiency in such processes and high performance in those drives (Robot arm, Conveyors). The direct current motor is the prime mover actuator in many high performance drives. This makes effective control of the high performance drives to wholly depend on the control of the DC motor. The angular speed and position are the two outputs of a DC Servomotor that can be controlled. If there is any error in the angular velocity or angular displacement profiles of those drives, they will miss their track or target. The resultant effect is always disastrous, causing huge wastes, loss of revenue, reduced output and sometimes human injury etc. Target position precision is therefore paramount in order to achieve high performance. There are existing conventional and intelligent controllers for achieving high performance through the control of the prime mover actuator (DC motor) but many of them have major drawbacks such as inability to minimize the control error to minimal acceptable value within the least possible time and their inability to accommodate nonlinearities (Backlash and Deadzone) associated with the plant etc. So, when high performance drives are considered, ANN that has the capability of handling these major drawbacks is paramount. Therefore, this work is aimed at characterization and development of ANN model of a PMDC motor.

\subsection{Theory}

DC motors are electromechanical actuator that converts electric energy into mechanical energy. The torque which is the rotational force developed on the motor shaft is directly proportional to the armature current and field flux (Kuo and Golnaraghi, 2002). To substantiate that, assume a current-carrying conductor being introduced into a magnetic field of flux $\phi$ (opentextbc.ca, 2016); if the distance of the conductor from the center of rotation is 
$\mathrm{r}$, then the relationship between the developed motor torque $\left(T_{m},\right)$, flux $(\phi)$, and current $\left(i_{a}\right)$ is; $K_{m} \phi i_{a}$ (Kuo and Golnaraghi, 2002) where $T_{m}$ is measured in N-M, $\phi$ in webers and $i_{a}$ in amperes whereas $k_{m}$ is the proportionality constant.

In addition to the torque developed, voltage known as back emf is generated across the DC motor terminals, when the conductor moves in the magnetic field. According to Kuo and Golnaraghi, (2002), this voltage is directly proportional to the shaft angular velocity, and tends to oppose the flow of current. The back emf $\left(e_{b}\right)$ and the shaft angular velocity are related by; $k_{m} \phi \omega_{m}$, where $\omega_{m}$ is the shaft velocity measured in $\mathrm{rad} / \mathrm{sec}$.

ANN can be applied in modeling of many control processes (Ramírez-Quintana, ChaconMurguia and Chacon-Hinojos, 2012). It is the choice of many designers because they can learn nonlinear input-output mapping from process data. Because of its learning ability, ANN, till moment, remains the alternative and probably the most effective way for modeling (Narendra and Parthasarathy, 1990). Perhaps, it has been emphasized that for successful NN modeling and control, complete controllability and observability of the process must be assumed (Saerens and Soquet,1991). More so Narendra and Parthasarathy, (1990), indicated that more work needs to be done in order to achieve more progress in nonlinear control theory.

\subsection{Implementation}

The first step in modeling a dynamic system is to fully define the system; the system can be separated into parts and modeled by subcomponents (MathsWork, 2012a). The Adaptive control system block diagram is shown in fig1, and modeling was done in line with the subcomponents.

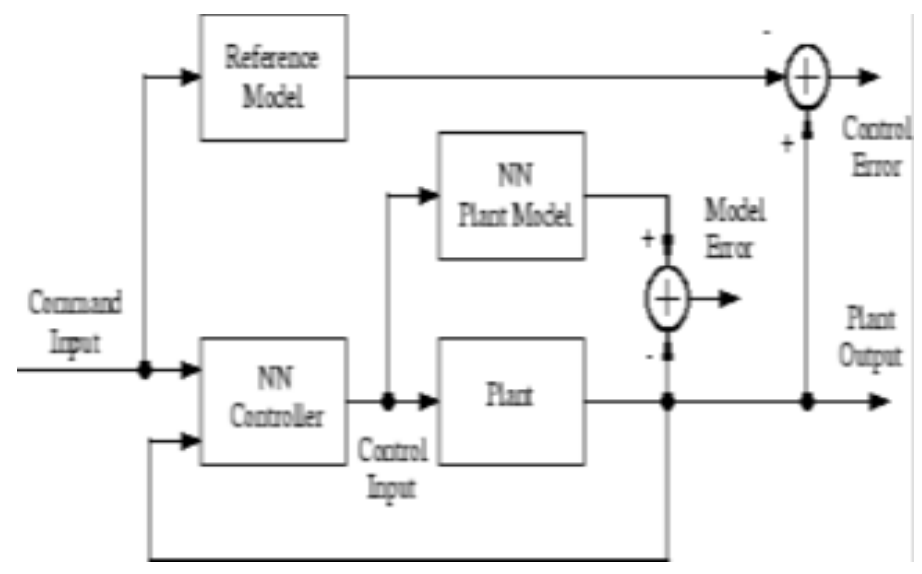

\section{Fig 1: The Adaptive Control System Block Diagram}

The neural model reference adaptive control architecture used two neural networks: a controller network and a plant model network. The plant model is identified first, and then the controller is trained so that the plant output follows the reference model output. The reference model provides the desired control system performance. It also helps the NN controller to avoid having the trajectory to be tracked changed too rapidly.

\subsection{Modeling and Characterization of a PM DC Motor}

Figure 2 shows the electromechanical representation of a DC motor, the diagram was used to develop the system level transfer function that characterized the behavior of the motor. 


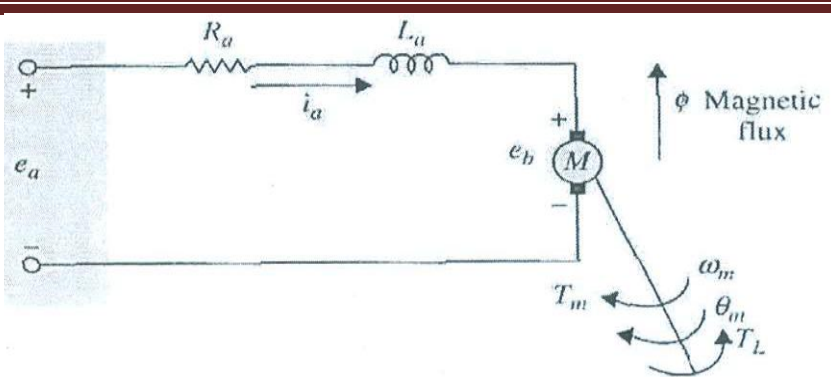

Fig 2: Electrical Model of DC Motor

The armature is modeled as a circuit with resistance $\mathrm{R}_{\mathrm{a}}$ connected in series with an inductance $\mathrm{L}_{\mathrm{a}}$ and a voltage source $\mathrm{e}_{\mathrm{a}}$ (Ananya, Aditya, Suneet ,2011; Scribd.com, 2011), and $\mathrm{e}_{\mathrm{b}}$ representing the back electromotive force (EMF) in the armature when the rotor rotates (Dhaval and Patel, 2015). From fig 2, it can be seen that the control of the DC motor is applied at the armature terminals in the form of applied voltage $e_{a}(t)$ (Ananya, Aditya, Suneet, 2011; Scribd.com, 2011). It can be deduced that the torque developed in the motor is proportional to the air-gap flux and the armature current, and equations that describe the motor behavior is stated by Kuo and Golnaraghi (2002) as:

$T_{m}(t)=K_{m}(t) \phi i_{a}(t)$

Since $\phi$ is constant, equation 1 is in form: $T_{m}(t)=K_{i} i_{a}(t)$

$K_{i} i_{a}(t)=i_{m} \omega_{m}+b \omega_{m}+T_{L}$

where, $\mathrm{k}_{\mathrm{m}}$ is a proportional constant and $T_{L}$ is load torque.

Considering the control input voltage $\mathrm{e}_{\mathrm{a}}(\mathrm{t})$ then, the cause and effect equations for the motor circuit are:

$$
\frac{d i_{a}(t)}{d t}=\frac{1}{L_{a}} e_{a}(t)-\frac{R_{a}}{L_{a}} i_{a}(t)-\frac{1}{L_{a}} e_{b}(t)
$$

$e_{b}(t)=k_{b} \frac{d \theta_{m}(t)}{d t}=K_{b} \omega_{m}(t)$

$\frac{d^{2} \theta_{m}(t)}{d t^{2}}=\frac{1}{J_{m}} T_{m}(t)-\frac{1}{J_{m}} T_{L}(t)-\frac{B_{m}}{J_{m}} \frac{d \theta_{m}(t)}{d t}$.

where the parameters are as defined (Basil, 2016; Scribd.com, 2016):

$i_{a}(t)=$ Armature current

$R_{a}=$ Armature resistance

$e_{b}(t)=$ Back emf

$T_{L}(t)=$ Load torque

$T_{m}(t)=$ Motor torque

$\theta_{m}(t)=$ Rotor displacement

$K_{i}=$ Torque constant in N-m/A.
$L_{a}(t)=$ Armature inductance

$e_{a}(t)=$ Applied voltage

$K_{b}=$ Back emf constant

$\emptyset=$ Magnetic flux in the air gap

$\omega_{\mathrm{m}}(\mathrm{t})=$ Rotor angular velocity

$J_{m}=$ Rotor inertia

$B_{m}=$ Viscous-friction coefficient

From equations (1) through (4), the applied voltage $e_{a}(t)$ is considered as the cause and equation (3) consider $\frac{d i_{a}(t)}{d t}$ the immediate effect due to the applied voltage. From equation (1), armature current $i_{a}(t)$ causes the motor torque $T_{m}(t)$, while in equation (4) the back emf 
$e_{b}(t)$ was defined. It can be seen also from equation (5) that the motor torque produced, causes the angular velocity $\omega_{m}(t)$ and displacement $\theta_{m}(t)$ of the rotor respectively.

The state variables of the system can be defined as: $i_{a}(t), \omega_{m}(\mathrm{t})$ and $\theta_{m}(t)$.

It is possible to eliminate all the non-state variables from equation (1) through (5) by direct substitution then, present the DC state equation in vector-matrix form as follows:

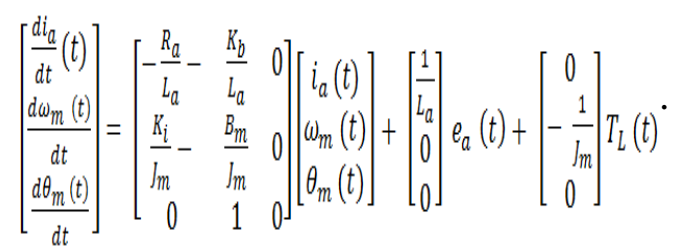

Note that in the case of equation $(6)$, that $T_{L}(t)$ is handled as a second input to the state equations. The transfer function between the motor displacement and the input voltage is obtained by applying Laplace transform to equations (4) and (5). The equations transformed to:

$$
\begin{aligned}
& j s^{2} \theta_{m}(s)+b s \theta_{m}(s)=k I(s) \\
& L s I(s)+R I(s)=E(s)-k s \theta_{m}(s)
\end{aligned}
$$

where $s$ is the Laplace operator. It can be deduced from equation (8) that transform of current is given as:

$$
I(s)=\frac{E(s)-k s \theta_{m}(s)}{R+L s}
$$

Substituting equation (8) into (9) yields:

$J s^{2}+b s \theta_{m}(s)=k \frac{E(s)-k s \theta_{m}(s)}{R+L s}$

The motor transfer function can be deduced by expanding and rearranging equation(10) as follows:

$$
\frac{\theta_{m}(s)}{E(s)}=\frac{k_{i}}{s\left[(R+L s)(J s+b)+k^{2}\right.}
$$

where $k=k_{i}$. Substituting for angular velocity in equation (11) yields:

$$
\frac{\omega_{m}(s)}{E(s)}=\frac{k_{i}}{(R+L s)(J s+b)+k^{2}}
$$

Equation (11) forms a single transfer function of the DC motor. Note that $T_{L}$ has been set to zero in equation (11). From the block diagram of figure 2, it can be seen how the transfer function is related to each block. It can be seen also from equation (11) that $s$ can be factored out of the denominator and the significance of the transfer function $\frac{\theta_{m}(s)}{E(s)}$ is that the DC motor is an integrating device between these two variables. The symbol $\theta_{m}(s)$ represents the rotor angular displacement, $E(s)$ is the input voltage and $\omega_{m}(s)$ is the transform of angular velocity respectively. 
The mathematical model of the DC motor has been developed but before the motor can be used, it is important to understand how the motor behaves before subjecting it to simulation and physical tests for measurement or operation. The DC motor is represented electromechanically by equating electrical loses from the left hand side of figure 2 to the mechanical forces on the right hand side. Since the single transfer function describing the motor is already derived in equation (11), it is important to determine the values of the variables (parameters) that were considered. The first parameter it's value was determined is the winding resistance of the motor $\left(\mathrm{R}_{\mathrm{a}}\right)$. This was determined by using the short and open circuit test. The rotor was locked in a particular position and voltages were applied to the terminals of the motor incrementally. The current increases and reaches a steady value. The value was recorded and the rotor position shifted to a new position. The same procedure was repeated, many values were recorded then, Ohm's law was used to determine the value of resistance at each point of rotor change then, the average of all the points were taken. The value of $R_{a}$ achieved is shown in table1.

The next parameter that was determined is the armature inductance $\left(\mathrm{L}_{\mathrm{a}}\right)$. This was achieved using an Inductance Capacitance Resistance (LCR) meter. The value was determined by setting the meter to "series inductance" and connecting the terminals of the motor to the input of the LCR meter. The rotor position was changed to different positions and readings were taken. The average values of the recorded inductances were taken. The $L_{a}$ value achieved is presented in table1. Another parameter value that was determined is the motor constant $\mathrm{K}$. This was determined by multiplication of the no-load current with the armature resistance to determine the voltage drop $\left(e_{b}\right)$ i.e. the back emf since the no-load current and speed were made available from the motor manufacturer through the datasheet. Thus, the motor constant was determined using the following speed-voltage equation:

$\omega_{m}=\frac{e_{a}-e_{b}}{k_{i}}$

where, $\omega_{m}$ is the rated speed of the motor in $\mathrm{rad} / \mathrm{sec}$. and $e_{a}$ is the rated input voltage. The value of $K$ and other parameters are shown in table 1 . The values of moment of inertia $(\mathrm{J})$ and damping coefficient (b) which are the mechanical forces governing the operation of the DC motor are subject to the system material properties and load characteristics. These values were deduced from the datasheet and compared with similar standard models of $200 \mathrm{~W}$, 200V, 1800 RPM DC motor ratings.

Table 1 DC Motor Parameter Values

\begin{tabular}{|cc|}
\hline Parameters & Values \\
\hline Moment of Inertia J & $0.0064 \mathrm{~kg}-\mathrm{m}^{2}$ \\
Damping Coefficient b & $0.001 \mathrm{Nm} / \mathrm{rad}^{-1}$ \\
Torque Constant Kt & $0.010 \mathrm{Nm} \mathrm{A}^{-1}$ \\
Electromotive force Constant Ke & $40 \mathrm{NmA}^{-1}$ \\
Electrical Resistance & $1.33 \Omega$ \\
Electrical Inductance & $0.05 \mathrm{H}$ \\
\hline
\end{tabular}

Using equation (11), and appropriate blocks from the Simulink toolbox, the Simulink model of the DC motor using speed as input is developed as shown in fig From the diagram, it can be seen that the motor has a built-in feedback loop caused by the back emf, $\mathrm{e}_{\mathrm{b}}$. The back emf physically represents the feedback of a signal that is proportional to the negative value of the speed of the motor. 


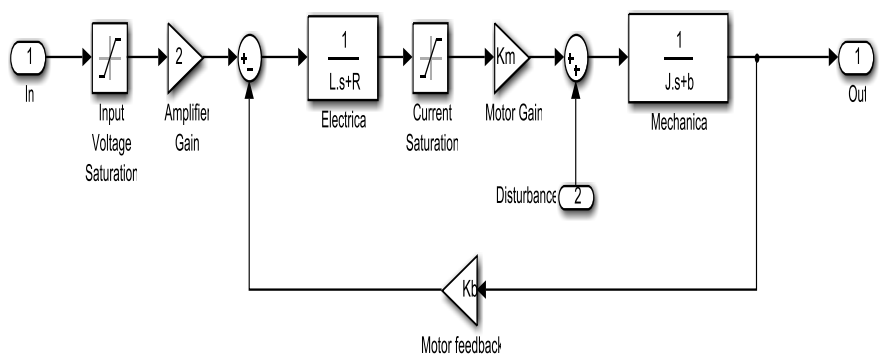

Fig 3: Simulink Model of a DC Servomotor in Terms of Speed

Focusing on equation (7), it can be noted that back emf constant $\mathrm{K}_{\mathrm{b}}$ represents an added term to the resistance $R_{a}$ and the viscous-friction coefficient $B_{m}$. Effectively, the back emf effect is equivalent to an electric friction which tends to improve the stability of the motor and invariably that of the system.

\subsection{Motor Equivalent Circuit in Discrete Form}

In order to simulate the ANN model of the motor in Simulink, there is need to construct an equivalent discrete time model of the motor. In line with that, the load torque was used since it is a second input to the state equations, and can be estimated as:

$\mathrm{T}_{\mathrm{L}}=\mu \omega^{2} m(t)\left[\operatorname{sgn}\left(\omega_{m}(t)\right)\right]$

(Weerasooriya and El-sharkawi, 1991) (14)

where $\mu=$ a constant, "sgn" or sign is the attribute of being positive or negative.

It is obvious that from equation (14), the load torque always opposes the direction of motion. The choice of load torque here is arbitral because considering load torque as one of the functions of a DC motor; it is a common characteristic for most propeller driven loads. Then using a sampling time interval of $\Delta T$, in discrete form the derivate of speed and current could be presented as follows:

$$
\begin{aligned}
& \frac{d \omega_{m}}{d T}=\frac{\omega_{m}(k+1)-\omega_{m}(k)}{\Delta T} \\
& \frac{d i_{a}}{d t}=\frac{i_{a}(k)-i_{a}(k-1)}{\Delta T} .
\end{aligned}
$$

where $k$ represents the time instant, then considering equations (15) and (16), the discrete equivalent of equations (2) and (3) will thus be:

$$
\begin{aligned}
& k_{i} i_{a}(k)=I_{m} \frac{\left[\omega_{m}(k+1)-\omega_{m}(k)\right]}{\Delta T}+b \omega_{m}(k)+T_{L}(k) \\
& k_{i} \omega_{m}(k)=-R_{a} i_{a}(k)-L_{a} \frac{\left[i_{a}(k)-i_{a}(k-1]\right.}{\Delta T}+v_{a}(k) \\
& i_{a}(k)=\frac{l_{m}}{(\Delta T) k_{i}}\left[\omega_{m}(k+1)-\omega_{m}(k)\right]+\frac{b \omega_{m}(k)}{k_{i}}+\frac{T_{L}(k)}{k_{i}} \\
& \frac{i_{a}(k)-i_{a}(k-1)}{\Delta T}=\frac{l_{m}}{k_{i}(\Delta T)^{2}}\left[\omega_{m}(k+1)-2 \omega_{m}(k)+\omega_{m}(k-1)\right]+\frac{b}{k_{i}(\Delta T)}\left[\omega_{m}(k)-\omega_{m}(k-1)\right]+\frac{1}{k_{i}(\Delta T)}\left[T_{L}(k)-T_{L}(k-1)(20)\right.
\end{aligned}
$$

Next, equations (19) and (10) were used in equation, after rearranging, the finite differential equation according to Weerasooriya and El-sharkawi, (1991), translates to: 


$$
\begin{aligned}
& \omega_{m}(k+1)=\alpha \omega_{m}(k)+\beta \omega_{m}(k-1)+\gamma \operatorname{sgn}\left(\omega_{m}(k)\right) \omega_{m}^{2}(k)+ \\
& \delta s g n\left(\omega_{m}(k-1)\right) \omega_{m}^{2}(k-1)+\xi_{a}(k)
\end{aligned}
$$

where $\alpha, \beta, \gamma, \delta$, and $\xi$ are:

$$
\alpha=\frac{-k_{i}{ }^{2}(\Delta T)^{2}+R_{a} I_{m} \Delta T-R_{a}\left(b(b \Delta)^{2}+2 L_{a} l_{m}-L_{a} b \Delta T\right.}{\left(L_{a} I_{m}+R_{a} l_{m} \Delta T\right)}
$$

$$
\begin{aligned}
& \beta=\frac{L_{a} b \Delta T-L_{a} I_{m}}{\left(L_{a} I_{m}+R_{a} I_{m} \Delta T\right)} \\
& \gamma=-\frac{\mu L_{a} \Delta T}{\left(L_{a} I_{m}+R_{a} I_{m} \Delta T\right)} \\
& \delta=\frac{\mu L_{a} \Delta T}{\left(L_{a} I_{m}+R_{a} I_{m} \Delta T\right)} \\
& \xi=\frac{\mu L_{a}(\Delta T)^{2}}{\left(L_{a} I_{m}+R_{a} I_{m} \Delta T\right)}
\end{aligned}
$$

$\alpha, \beta$, and $\xi$ are constant values based on the motor parameters $I, K, B, R_{A}$ and $L_{a}$ and sampling period $T$. The coefficients $\gamma$ and $\delta$ are functions of the above parameters and also a function of the frictional force $\mu$. $K$ on the other hand denotes $k^{\text {th }}$ instance i.e. time step. Since the mathematical model representing a single transfer function of a DC motor has been achieved in equation (11) then, in line with the objective of achieving the discrete form of the DC motor, equation (21) was rewritten to obtain the final discrete form of the DC motor as follows:

$v_{a}(k)=f\left[\omega_{m}(k+1), \omega_{m}(k), \omega_{m}(k-1)\right.$

Equation (27) is the developed discrete model of a DC motor in terms of speed. The term in the right hand side of equation 27 is a nonlinear function of the speed $\omega_{m}$ which is equal to:

$\frac{1}{\xi}\left[\begin{array}{c}\omega(k+1)-\alpha_{\omega}(k)-\beta_{\omega}(k-1)-\gamma \operatorname{sgn}\left(\omega_{m}(k)\right) \omega_{m}^{2}(k) \\ -\delta \operatorname{sgn}\left(\omega_{m}(k) \omega_{m}^{2}(k-1)\right.\end{array}\right]$

The $f$ in the above expression is the unknown function which is nonlinear in nature. ANN is used for controlling the unknown variables of the motor. Thus the $\mathrm{NN}$ will be trained to identify this unknown nonlinear function. $v_{a}(k)$ is a function of speed at successive time intervals $k+1, k$ and $k-1$. Therefore, the following forms the input speed to the ANN:

$\omega_{m}(k+1) \rightarrow$ expected speed at next instance

$\omega_{m}(k) \rightarrow$ present speed

$\omega_{m}(k-1) \rightarrow$ speed at previous instance

$\omega_{m}(k+1), \omega_{m}(k)$ and $\omega_{m}(k-1)$ are independent parameters or variables of the nonlinear function $(f$.). 
4.0 Modeling of the DC Motor in Terms of Angular Displacement $\left(\theta_{\mathrm{m}}\right)$

From reviews, it is obvious that precise control of speed does not mean accurate control of the position. So for accurate position to be achieved, position profile should be incorporated in the equation governing the precise control of the speed by simple integration. The block diagram for the integration is shown in fig 4.

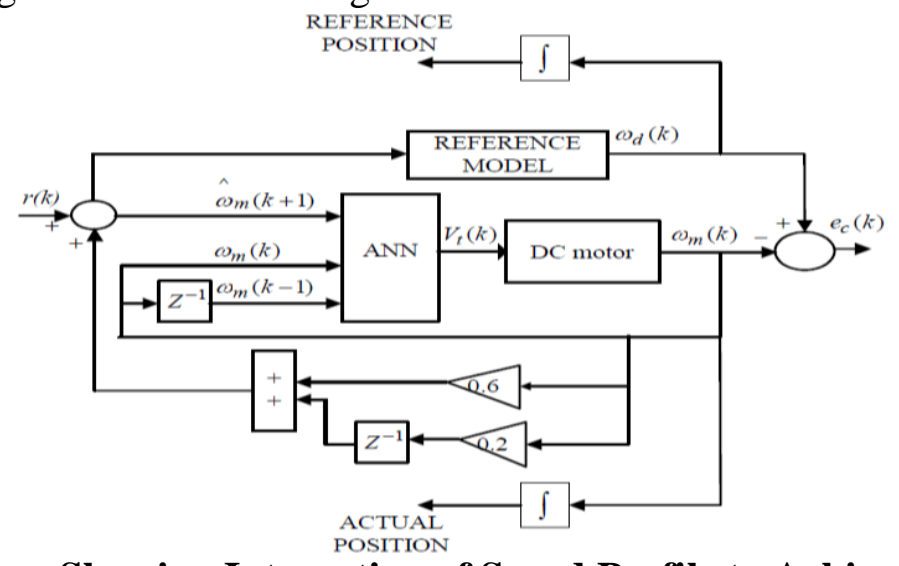

Fig 4: Block Diagram Showing Integration of Speed Profile to Achieve Position

Then to improve the performance of the position control, a feedback module was incorporated into the network. The position error is amplified through the feedback module and effectively, used to modify the actual motor speed $\omega_{m}(k)$. Then, from equation (21),

$$
\begin{aligned}
& v_{a}(k)= \\
& \frac{\omega_{m}(k+1)-\alpha \omega_{m}(k)-\beta \omega_{m}(k-1)-\gamma \operatorname{sign}\left(\omega_{m}(k)\right) \omega_{m}{ }^{2}(k)-\delta \operatorname{sign}\left(\omega_{m}(k-1)\right) \omega_{m}^{2}(k-}{\xi} .
\end{aligned}
$$

From reviews also, it is known that the load affects the voltage through its inertial and noninertial terms. The inertial term contributes to the linear variation of the voltage with speed while the non-inertial terms contribute to the non- linear variation of voltage. These effects due to non-inertial terms are small compared with the effects due to the inertial terms. If the effects due to the non-inertial terms are neglected in equation (29), from the work of Weerasooriya and El-Sharkawi, (1991), the equation becomes:

$v_{a}(k) \propto\left[\omega_{m}(k+1)-\alpha \omega_{m}(k)-\beta \omega_{m}(k-1)\right]$.

Since

$$
\omega_{m}(k+1), \omega_{m}(k), \omega_{m}(k-1)
$$

are speeds at successive intervals, and

$$
\left|\omega_{m}(k+1)-\omega_{m}(k)\right| \ll \omega_{m}(k+1)
$$

Then, it can be assumed that the Equation:

$\omega_{m}(k+1) \approx \omega_{m}(k-1)=\omega_{m}(k)$.

$v_{a}(k) \propto(1-\alpha-\beta) \omega_{m}(k)$.

Therefore, $(1-\alpha-\beta)=1-\frac{-k_{i}^{2}(\Delta T)^{2}+R_{a} I_{m} \Delta T-R_{a}(b \Delta)^{2}+2 L_{a} I_{m}-L_{a} b \Delta T}{\left(L_{a} I_{m}+R_{a} I_{m} I T\right)}-\frac{L_{a} b \Delta T-L_{a} I_{m}}{\left(L_{a} I_{m}+R_{a} I_{m} \Delta T\right)}$ 
After the subtraction in equation (30), the equation becomes;

$v_{a}(k)=\frac{k_{i}^{2}(\Delta T)^{2}+R_{a}(b \Delta)^{2}}{\left(L_{a} I_{m}+R_{a} I_{m} \Delta T\right)}$

Note that the terms in the right side of equation (34) are positive. Effectively it means that $(1-\alpha-\beta)$, is positive as well. Then, if that is the case, it means that $v_{a}(k)$ increases with increase in motor speed $\omega_{m}(k)$. Direct substitution can be used to substitute for position in equations (2), (3) and (14) i.e. rewriting the angular velocity $\omega_{m}(k)$ in terms of angular displacement $\theta_{m}$, which amounts to the position. The Simulink model is constructed as shown in figure 5 .

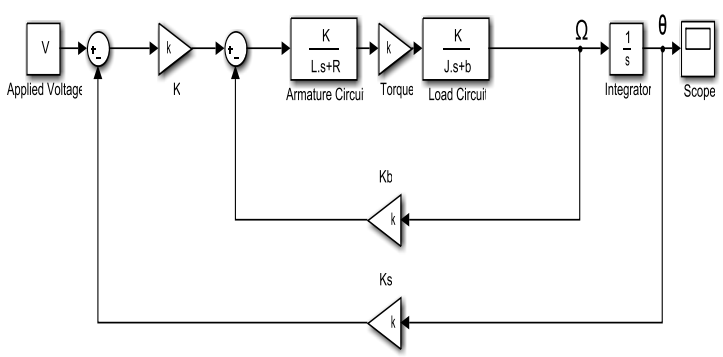

Fig 5: Simulink Model of a DC Servomotor in Terms of Speed and Position

Then the equations yield:

$$
\begin{aligned}
& k_{i} \theta_{m}=-R_{a} i_{a}-L_{a} \frac{\partial i_{a}}{\partial t}+v_{a} \\
& k_{i} i_{a}=I_{m} \theta_{m}+b \theta_{m}+T_{L} \\
& T_{L}=\mu\left(\frac{d \theta_{m}}{d t}\right)^{2}\left[\operatorname{sgn}\left(\theta_{m}\right)\right] .
\end{aligned}
$$

Next is to estimate the derivatives of position and current in discrete form using a sampling interval of $\Delta T$ and forward difference.

$$
\begin{aligned}
& \frac{d \theta_{m}(k)}{d t}=\frac{\theta_{m}(k+1)-\theta_{m}(k)}{\Delta T} . \\
& \frac{d^{2} \theta_{m}(k)}{d t^{2}}=\frac{\frac{d \theta_{m}(k+1)}{d t}-\frac{d \theta_{m}(k)}{d t}}{\Delta T} . \\
& \frac{d^{3} \theta_{m}(k)}{d t^{3}}=\frac{\frac{d^{2} \theta_{m}(k+1)}{d t^{2}}-\frac{d^{2} \theta_{m}(k)}{d t^{2}}}{\Delta T} \\
& \frac{d i_{a}}{d t}=\frac{i_{a}(k+1)-i_{a}(k)}{\Delta T} . \\
& T_{L}(k)=\mu\left(\frac{d \theta_{m}(k)}{d t}\right)^{2}\left[\operatorname{sgn}\left(\theta_{m}(k)\right] .(43)\right.
\end{aligned}
$$


$\frac{d T_{L}(k)}{d t}=\frac{T_{L}(k+1)-T_{L}(k)}{\Delta T}$.

Next is to evaluate the armature current $i_{\boldsymbol{a}}$ in terms of angular displacement $\theta_{m}$ which translates to position here. Then substituting $\boldsymbol{i}_{\boldsymbol{a}}$ in terms of $\theta_{m}$ using equations (38) into (37) gives:

$k_{e} \dot{\theta}_{m}=-\frac{R_{\underline{a}}}{k_{e}}\left[I_{m} \ddot{\theta}_{m}+b \dot{\theta}_{m}+T_{L}\right]-\frac{L_{l}}{k_{e}}\left[l_{m} \ddot{\theta}_{m}+b \ddot{\theta}_{m}+T_{L}\right]+v_{a}$

Or

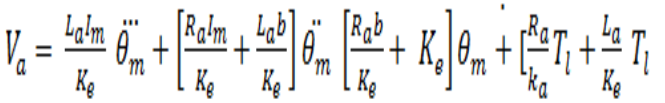

Integrating equations (39), (40), (41), (42), (43) and (44) into equation (46) then the input voltage in equation (40) can be written as a function of:

$\theta_{m}(k+1), \theta_{m}(k), \theta_{m}(k-1)$ and $\theta_{m}(k-2)$.

$v_{a}(k)=g\left[\theta_{m}(k+1), \theta_{m}(k), \theta_{m}(k-1), \theta_{m}(k-2)\right]$

Equation (47) is the developed mathematical model of the motor in terms of position. Effectively, equation (47) forms the relationship between the input voltage $v_{a}$ and the motor position $\theta_{m}$ at four successive sampling instances. Assume that the term $\theta_{m}(k+1)$ is replaced in equation (47) with desired reference motor position at next instance as $\boldsymbol{\theta}_{d}(k+1)$, and the control voltage (the input voltage) $v_{a}(k)$ computed with the following equation, then;

$v_{a}(k)=g\left[\theta_{d}(k+1), \theta_{m}(k), \theta_{m}(k-1), \theta_{m}(k-2)\right] .(48)$

Hence, the computed voltage $v_{a}(k)$, at sampling instance $k$ is applied, and then the resulting motor position at instant $(k+1)$ is given as:

$\theta_{m}(k+1)=\theta_{d}(k+1)$

Therefore, the desired motor position effectively takes the following forms of input to the ANN:

$\theta_{d}(k+1) \rightarrow$ the reference position

$\theta_{m}(k) \rightarrow$ Position at first instance

$\theta_{m}(k-1) \rightarrow$ Position at second instance

$\theta_{m}(k-2) \rightarrow$ Position at third instance

\subsection{ANN Model of DC Motor}

Before ANN can be used to control the operation of the DC motor, the DC motor being the plant must also be modeled using ANN tool. From equation (27) where $v_{a}(k)$ is a function of speed at successive time intervals $\mathrm{k}+1, \mathrm{k}$ and $\mathrm{k}-1$ for any required trajectory, what happens is that the ANN Inverse Model (AIM) generates an output that is proportional to the voltage required at the input of the DC motor to produce these speed at the time intervals. Here, the input-output mapping is many to one. Perhaps, disturbances and other uncertainties may lead 
to the input-output mapping to become one-to-many leading to reduction in the control performance of the system i.e. degradation. Though, the AIM relies on the accuracy of the model used for the controller design, this work will not worry about the degradation. The block diagram of the speed based AIM is shown in figure 6 .

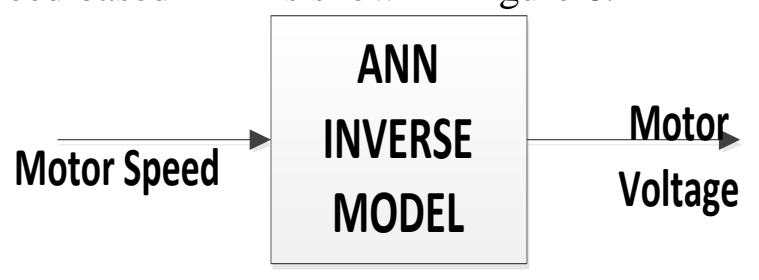

Fig 6: Block Diagram of the AIM

Structure of the AIM: The concept of inverse model is based on the principle that the system output depends on the preceding output and input values. The AIM for this work is made up of three inputs and a single output structure for the three successive speed instances. Based on inverse model as seen from equation (27), the three inputs are $\omega_{m}(k+1)$; Speed at first instance $\omega_{m}(k)$; speed at second instance, $\omega_{m}(k-1)$; speed at third instance and the output is the $V_{a}(k)$ which is the motor terminal output voltage $V_{t}(k)$ from fig

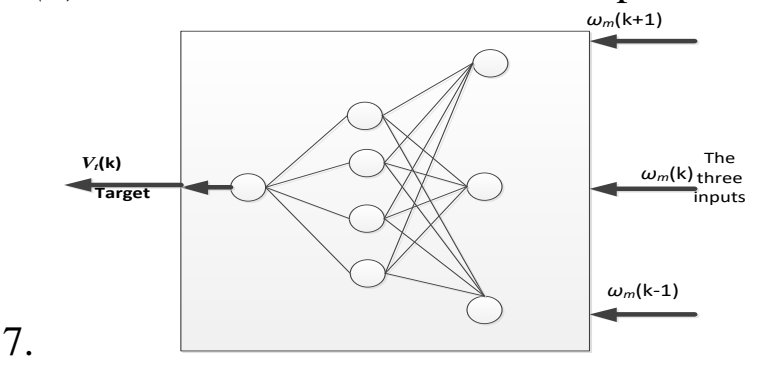

Fig 7: Structure of the AIM

Therefore, based on same equation (27), the nonlinear function $(f$.) can be presented in the following form:

$$
\begin{aligned}
& f\left(\omega_{m}(k+1), \omega_{m}(k), \omega_{m}(k-1)=\right. \\
& \underline{\left(\omega_{m}(k+1)-\omega_{m}(k)-\beta \omega_{m}(k-1)-\gamma \operatorname{sgn}\left(\omega_{m}(k)\right) \omega_{m}^{2}(k)-\delta \operatorname{sgn} n\left(\omega_{m}(k-1)\right) \omega_{m}^{2}(k-1)\right.}
\end{aligned}
$$

The values of $\left(\omega_{m}(k+1), \omega_{m}(k)\right.$, and $\omega_{m}(k-1)$ apparently form the independent inputs of the ANN and the corresponding output as well is generated from equation (49).

\subsection{Performance Evaluation of the AIM}

The generated $\left(\omega_{m}(k+1), \omega_{m}(k), \omega_{m}(k-1)\right.$ inputs and the corresponding targets $V_{a}(k)$ are used for offline training of the AIM to represent any DC motor with unknown parameters. From figure 7, it could be seen that the performance error is represented by $e_{i}(k)$. In evaluating the AIM performance, the value of $\left[e_{i}(k)\right]^{2}$ for all $k T$ that are elements of time from 0 to $t_{\mathrm{f}}$ is minimized, that is (Weerasooriya and El-Sharkawi, 1991);

\section{$\left[e_{i}(k)\right]^{2} \forall k T \in\left[0, t_{f}\right]$}

where $T$ is the sampling period and $t_{f}$ is the time for which simulation is performed. Sampling time of $0.04 \mathrm{sec}$ was used. The terminal voltage (estimated) is given by: 
$v_{\text {text }}(k-1)=N\left[\omega_{m}(k), \omega_{m}(k-1), \omega_{m}(k-2)\right](52)$

Once the DC motor is excited by an input signal, the output from the DC motor which is the motor speed $\omega_{m}$ is fed into the AIM as an input. The terminal voltage is compared with the actual motor output $e_{o}(k)$ for a common excitation signal. Then the mean square value of the error $e_{i}(k)$ between the actual motor input and the estimated output voltage yields the performance error of the AIM. The performance evaluation is illustrated in fig. 8.

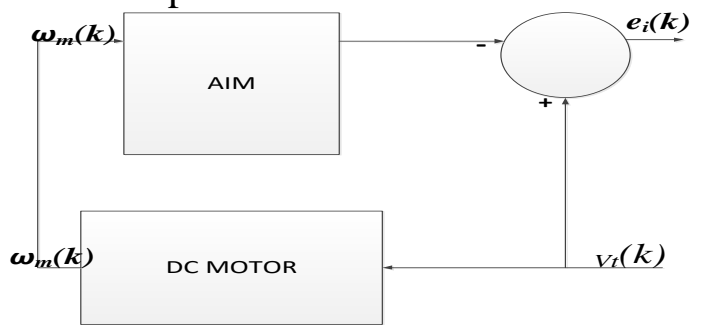

Fig 8: Performance Evaluation of AIM

\subsection{Structure of The Position-based AIM}

From equation (49), the input variables at four successive instances form the input to the ANN and the terminal voltage $V_{T}(k)$ corresponds to the desired output of the controller that is fed into the motor. To obtain the training sets, sequence of voltage signals capable of exciting the motor are applied and the motor position at successive sampling instants recorded then, the training set can be generated from those recorded input and output data. So, each training set is made up of the four successive motor positions and the applied voltage. The four successive instances are $\theta_{d}(k+1)$; the reference position, $\theta_{m}(k)$; position at first instance, $\theta_{m}(k-1)$; Position at second instance, $\theta_{m}(k-2)$; position at third instance and the applied voltage $V_{a}(k)$.The structure of position based AIM is as shown in fig 9 .

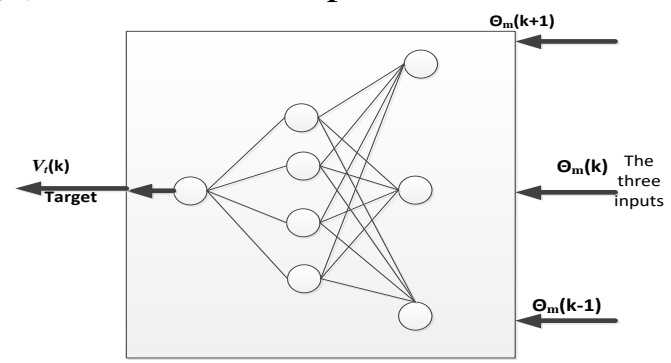

Fig 9: Structure of Position-based AIM

\section{1: Performance Evaluation of the Position-based AIM}

The training of the ANN was performed as discussed in subsection 6.2. To evaluate the performance of the position-based AIM, the parameter that is most important to be considered is the modeling error. This error is recorded in the form of: $\left[e_{i}(k)\right]^{2} \forall k T \in\left[0, t_{f}\right]$ (Weerasooriya and El-Sharkawi, 1991)

where $T$ is the sampling period and $t_{f}$ is the time for which simulation will be performed. The terminal voltage (estimated) is given by:

$\hat{V}_{\mathrm{t}}(\mathrm{k}-1)=\mathrm{N}\left[\widehat{\theta}_{\mathrm{m}}(\mathrm{k}), \theta_{\mathrm{m}}(\mathrm{k}-1), \theta_{\mathrm{m}}(\mathrm{k}-2)\right] \quad($ Weerasooriya and El-Sharkawi, 1991)

Hence, those randomly-generated input variables at four successive instants and the corresponding output are the parameters that will be used in offline training of the ANN. The 
block diagram for the performance evaluation of the AIM with position as input is shown in fig 10 .

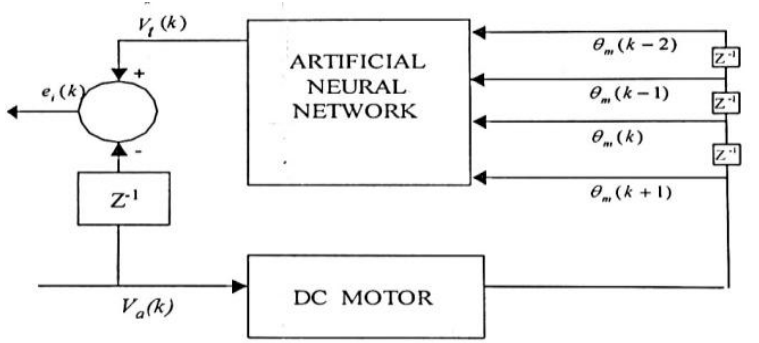

Fig 10: Performance Evaluation of the AIM with Position as Input

\section{0: Speed and Position Based MRAC System}

The essence of establishing the evaluation performance of the AIM is to minimize modeling error and once that is done, the position control can be accomplished by incorporating the AIM with the Model Reference Adaptive Control mechanism as shown in fig 11. The work of this MRAC is to generate a reference position $\theta_{\mathrm{d}}(\mathrm{k})$ that will serve as a reference point to the applied voltage $V_{t}(k)$ generated by the AIM to drive the motor position to $\theta_{\mathrm{m}}(\mathrm{k})$ as shown in fig 11.

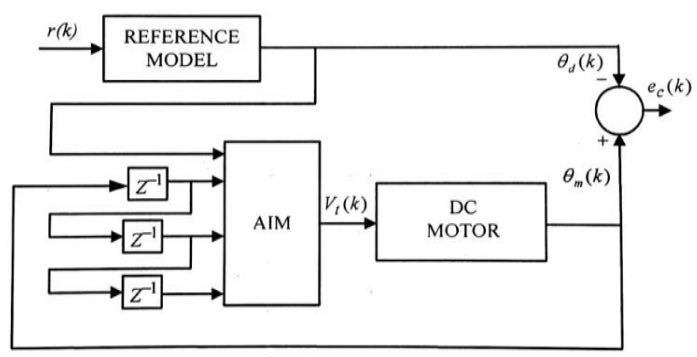

Fig 11: Block Diagram for Position Control of DC Motor in Simulink

As presented in equation (61), the terminal voltage is calculated so as to bring down the error to a minimal; such that $\left[e_{i}(k)\right]^{2} \forall k T \in\left[0, t_{f}\right]$ is minimized. The reference position generated by the MRAC system should be within the physical dynamic capabilities of the motor. Second-order reference model is deployed so that the dynamic parameters of the motor can be varied. From figure 12, the $r(k)$, serves as the input to the system and the second-order reference model takes the form of:

$\theta_{d}(k+1)=0.6 \theta_{d}+0.2 \theta_{d}(k-1)+r(k)$ (Weerasooriya and El-Sharkawi, 1991) (55)

To achieve a successful training of the network, it will be more realistic if the past values of $\theta_{\mathrm{d}}(\mathrm{k})$ from equation (55) be replaced with past values of the actual current output $\theta_{\mathrm{m}}(\mathrm{k})$ in order to avoid arriving at unpredictable results. By so doing, the new estimate for the desired output could then be $\theta_{\mathrm{m}}(k+1)$. Therefore, the terminal voltage from the AIM controller will be generated by using the positions of the motor at four successive instants with the estimated output in the form:

$\hat{V}_{\mathrm{t}}(\mathrm{k}-1)=\mathrm{N}\left[\widehat{\theta}_{\mathrm{m}}(\mathrm{k}), \theta_{\mathrm{m}}(\mathrm{k}-1), \theta_{\mathrm{m}}(\mathrm{k}-2)\right]$

Ranges of input $r(k)$ to the system, $\theta_{\mathrm{m}}(\mathrm{k}), \theta_{\mathrm{m}}(\mathrm{k}-1)$ and $r(k)$ are used to generate the desired reference position from the MRAC at instant $(\mathrm{k}+1)$ using equation (52). Consequently, the desired reference positions and the motor positions at the $\mathrm{k}, \mathrm{k}-1, \mathrm{k}-2$ instants will form the 
input to the AIM. Figure 12 is the block diagram of the control scheme with position as input. So it is the duty of the AIM to evaluate the voltage at the instant $(k+1)$, which is the voltage expected to drive the motor in accordance with the reference position.

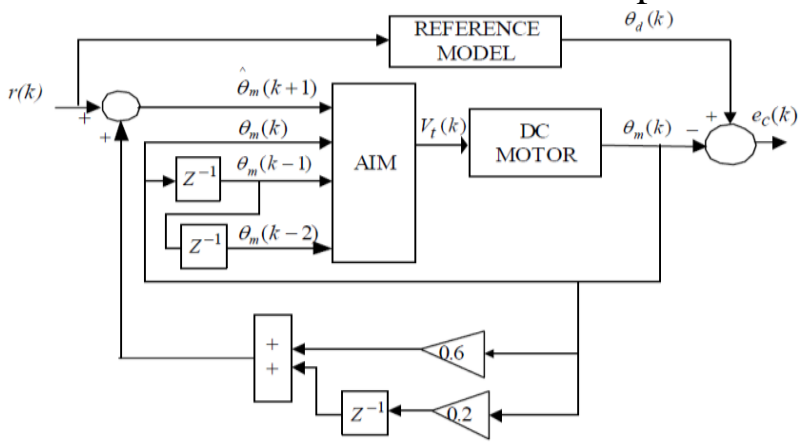

Fig 12: Block Diagram Showing Position Control System for DC Motor in Simulink

The essence of this work is to develop a model that will improve performance. To improve the position control performance, the speed and position profile were integrated together to form a single control system as shown in figure 13 . When the motor position $\theta_{m}$ is less than the desired position $\theta_{d}$, the AIM speed input ${ }_{\omega}^{*} m(k)$ is increased. This increment effectively brings about an increase in the terminal output voltage $V_{t}(k)$ of the AIM. The increase in terminal output voltage apparently increases the output speed $\omega_{m}(k)$ of the DC motor and thereby, reducing the position error. So the amplified position error modifies the speed input as follows:

$$
{ }_{\omega}^{*} m(k)=\omega_{m}(k)+K_{p}\left(\theta_{d}-\theta_{m}\right) \quad(57){ }_{\omega}^{*} m(k+1)=0.6{ }_{\omega}^{*} m(k)+0.2{ }_{\omega}^{*} m(k-1)+r(k)
$$

(Weerasooriya and El-Sharkawi, 1991) (58)

Equation (57) forms the mathematical model for the developed S and P based control system for motor.

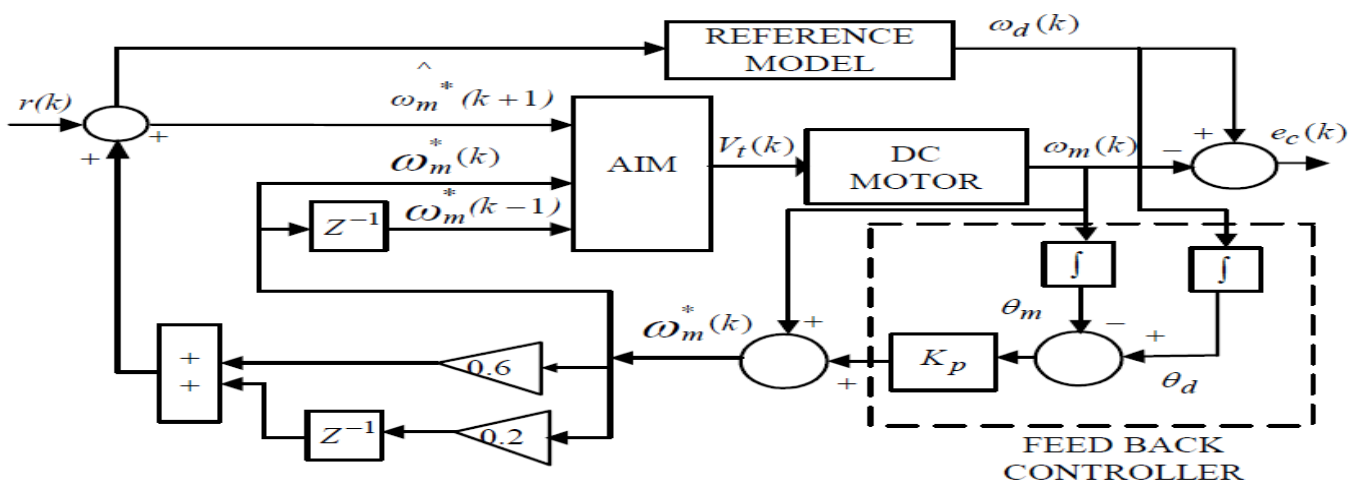

Fig 13: Speed and Position Based Control System Model in Simulink

\section{Conclusion}

The parameters of the PM DC motor were successfully characterized and the mathematical model representing a single transfer function of the DC motor developed hence, the discrete form of the motor. The model was developed in two instances. In the first instance, the motor speed was used as input to the AIM while the position was used as input in the second instance. From previous research works, using speed as input to the AIM improves accurate speed control but not precise position control. Perhaps, integration of speed and position 
profiles yields the Speed and Position inverse model of fig 13 that ensures accurate speed and precise position control.

\section{Reference}

Ananya R., Aditya G., and Suneet S.(2011). MATLAB based real time control implementation of DC servo motor using PCI card: Thesis paper, Retrieved from

Balduzzi D., McWilliams B., Butler-Yeoman T. (2017). Neural Taylor Approximations: Convergence and Exploration in Rectifier Networks; ICLR 2017 conference: Retrieved from https://openreview.net/forum?id=HyWG0H5ge

Basil H. (2016). Control Systems: Modeling in the Frequency Domain. Retrieved from https://www.scribd.com/presentation/290141743/Modeling-in-the-Frequency-Domain.

Dhaval M., and Patel K. (2015). Mathematical Modeling of Open Loop Pmdc Motor Using Matlab/Simulink: International Journal of Engineering Development and Research (www.ijedr.org), Volume 3, Issue 1.

Hagan M. T., Demuth H. B. (2015). Networks for Control: Retrieved from https://pdfs.semanticscholar.org/362b/a346b7e530e0bb22977fb4c236f20ea652bd.pdf.

Hagan M.T., and Demuth H.B. (2002). An introduction to the use of neural networks in control systems: International Journal of Robust and Nonlinear Control Volume12, Issue11, Pages 959985

MathsWork.com. (2017). Design Neural Network Predictive Controller in Simulink: Retrieved from https://www.mathworks.com/help/nnet/ug/design-neural-network-predictive-controller-insimulink.html.

Scribd.com. (2017). Model Reference Control: Control Systems (Neural Network Toolbox). Retrieved from https://www.scribd.com/document/59691396/mrefrobotarm.

Valluru B.R.(1995). C++ Neural Networks and Fuzzy Logic. MTBooks, IDG Books Worldwide, Inc. ISBN:n1558515526.

Weerasooriya S. and El-Sharkawi M.A. (1991). Identification and control of a DC motor using Backpropagation Neural Networks: IEEE Transactions on Energy Conversion, Vol.6, No.4, pp. 663669.

Zurada and Jacek M., (1992). Introduction to Artificial Neural Systems: Publisher PWS Publishing Company, 1992. ISBN: 053495460X, 9780534954604. 